\title{
A LOW COMPLEXITY SPECK-BASED CODEC FOR MULTISPECTRAL FLUORESCENCE MICROSCOPIC IMAGES
}

\author{
Awais Mansoor* \\ School of Electrical and Computer Engineering \\ Purdue University \\ 465 Northwestern Ave. \\ West Lafayette, IN 47907, USA.
}

\author{
J. Paul Robinson, Bartek Rajwa \\ Bindley Bioscience Center \\ Purdue University \\ 1203 West State Street \\ West Lafayette, IN 47907, USA.
}

\begin{abstract}
The distortion scalable wavelet based coder SPECK is widely referred to in literature as achieving excellent coding performance with very low computational complexity compared to other state-of-the-art encoders. In this paper, we propose a 3D extension to SPECK designed for lossless coding of lowSNR fluorescence microscopic multispectral images by joining and encoding together different wavelet subbands. The idea is based on an observation that in comparison to other image types, fluorescence images have a larger proportion of energy concentrated in the lowest frequency subbands, whereas the energy inside higher frequency subbands constitute a considerably small portion. Also, subbands at the same level of wavelet-transformed image are found to be highly correlated. Consequently, encoding higher subbands by grouping them together in a single block results in a considerable bit saving. In order to maximize the block size of coefficients that can be encoded together, a new set type is introduced. The proposed technique applied to test multispectral microscopic images demonstrates a significant bit savings.
\end{abstract}

Index Terms- 3D SPECK, fluorescence imaging, multispectral imaging, microscopic image encoding, low-SNR image encoding.

\section{INTRODUCTION}

Optical molecular imaging provides a non-invasive measurements of biological processes in living organisms. A key technique for molecular imaging is fluorescence microscopy, and its advanced version, multispectral (multiband) fluorescence microscopy (MFM). MFM techniques are being widely used to study cell cycle, gene expression, dynamic interactions among cells and cellular components, signaling pathways, and many other cellular processes [1]. However, imaging of live cells or tissues is difficult owing to possible cytotoxicity of fluorescent labels and phototoxicity of the imag-

\footnotetext{
* The authors thank Richard Levenson (Cambridge Research \& Instrumentation, Inc., Woburn, MA), for providing test images for this research. Address all correspondence to A. Mansoor, amansoor@purdue.edu
}

ing process. Therefore, the quality of acquired images is determined by limited excitation power, as well as the limited amount of fluorescent labels which can be used in live-cell conditions. In consequence, the photon budget in live cell imaging is usually poor. Owing to the limited number of photons which can be collected, the photon count per pixel in live-cell MFM is usually quite low. This fact as well as the presence of noise (photon noise, Fano noise, and fixed pattern noise of the detectors) means that the collected digital images suffer from a low SNR [2].

Multispectral imaging has broader applications than MFM. Due to smoothness of spectral response a very high correlation exists between different bands of MFM images. Discrete wavelet transform (DWT)-based methods have proven quite successful in exploiting correlation-related redundancies. Several DWT-based techniques have been developed during the last decade, and the most successful of them are codecs based on Shapiro's EZW [3] and its refinement by Said et al. as SPIHT [4]. Pearlman et al. proposed a low-complexity block-based alternative called SPECK [5] . The algorithm shows compression performance comparable to SPIHT with faster encoding compared to both SPIHT and EBCOT for 2D images [6]. Extension of 2D imagecompression techniques to $3 \mathrm{D}$ has been investigated in the literature for a wide range of applications. In particular, Kim et al. have proposed a modified SPIHT to compress video sequences [7]. Dragotti et al. applied wavelet transform along spatial axes and KLT along the spectral axis before coding the coefficients by SPIHT algorithm [8]. Pearlman et al. proposed an extension to block-based low-complexity SPECK by encoding the inter-band redundancies exploited by three-dimensional wavelet transform [9]. Finally, Khelifi et al. demonstrated extensions of 2D SPIHT and 2D SPECK encoders to jointly encode two consecutive bands that are independently decomposed using 2D wavelet transform [10]. Their method, although reduces the computational complexity, also limits the algorithm's ability to exploit interband redundancies.

Herein we present a new lossless low-complexity SPECKbased algorithm designed for MFM images. The key idea is 
to exploit the interband redundancies of these images by encoding the maximally correlated wavelet subbands as a single block. The blocks are split to maximize the intra-block correlation within the newly formed children blocks. The method has been demonstrated to provide significant improvement over state-of-the-art coders such as SPIHT, SPECK, and 3DSPIHT, while maintaining modest computational complexity.

\section{THE SPECK ALGORITHM: AN OVERVIEW}

The section provides a brief summary of the original 2D SPECK algorithm proposed by Pearlman et al. [5]. Let $\chi$ be an adequately wavelet-transformed $2 \mathrm{D}$ image represented by an indexed set of coefficients $\left\{c_{i, j}\right\} . \quad \chi$ is defined to exhibit hierarchal pyramidal structure characterized by different levels of decomposition. The algorithm maintains a set of three lists: list of significant pixels (LSP), list of insignificant pixels (LIP), and list of insignificant sets (LIS). At any stage, LIS contains the sets of type $S$ of different sizes that have not yet been found to be significant. LSP contains coefficients/sets found to be significant. If a set $S$ of size $1 \times 1$ is found significant in LIS, its sign is encoded and the block is moved to LSP. Sets of type $I$ are similarly evaluated against the current threshold; if the set is found to be significant it is split into three sets of type $S\left(S_{1}, S_{2}\right.$, and $\left.S_{3}\right)$ and a set of type $I$. The newly formed $S$ sets are evaluated against the current threshold $n$, and the significant subsets are iteratively partitioned into subsets of 4 , while insignificant subsets are moved to LIS. Finally, during the refinement pass the $n$-th most significant bit of each entry in LSP is encoded, except those coefficients that have been added to the list during the last sorting pass.

\section{PROPOSED LOW-COMPLEXITY 3D SPECK: THE METHODOLOGY}

A strong correlation that exists among different spectral bands of MFM images can result in a huge bit savings in both storage and transmission. Wavelet transform-based techniques have been found very useful in effectively exploiting correlation-based redundancies. Our method exploits these redundancies by collectively encoding correlated wavelet subbands at the same level of decomposition as a single block. The idea is motivated by the fact that a great proportion of energy inside a 3D wavelet-decomposed image is concentrated in the lowest-frequency subband cube. In addition, the number of significant coefficients in higher frequency subbands is sparse, and significant coefficients in these subbands are concentrated in a closed neighborhood. In order to ensure that the maximum number of insignificant coefficients are encoded together, in addition to type- $I$ and type- $S$ of traditional SPECK, an intermediate type $Y$ is introduced that divides subbands at the same level of decomposition into two subblocks instead of splitting them into individual subbands. These intermediate $Y$-type sets address redundancies in those block subbands where significant coefficients are sparse and concentrated in fewer subbands in a closed neighborhood.

Let $\chi$ be an adequately wavelet-transformed multispectral image represented by an indexed set of coefficients $\left\{c_{i, j, \lambda}\right\}$. The proposed algorithm divides $\chi$ into sets of three types: sets of type $S$ forming the root of the pyramid, sets of type $I$ obtained by chopping off $S$ sets from $\chi$, and intermediate sets of type $Y$ obtained through the unequal disseverance of significant $I$ sets. Before elaborating on the methodology, let us define a size measure for $Y$-type sets to be the number of wavelet subbands inside a $Y$ set: $\operatorname{size}\left(Y_{m}\right)=C\left(Y_{m}\right)=$ $\left|Y_{m}\right|$. $\left|Y_{m}\right|$ can be either 3 or 4 . Let us also define an indicator function to mark the significance of a block of coefficients. A set of coefficients is considered significant at the $n$-th threshold level if for any set of pixels $\mathbf{T}$

$$
\gamma_{n}(\mathbf{T})= \begin{cases}1 & \text { if } 2^{n} \leq \max \left|c_{i, j, \lambda}\right| \in \mathbf{T} \\ 0 & \text { otherwise }\end{cases}
$$

The algorithm consists of four steps: the initialization step, the sorting pass, the refinement pass, and the quantization step. The pseudocode for the new 3D-SPECK algorithm is presented in Algorithm 1. The pseudocode calls three functions, Process $S\left(S_{m}\right)$, ProcessI $\left(I_{m}\right)$, and Process $Y\left(Y_{m}\right)$, that are detailed in Algorithm 1. Sets $S$ are treated in the same way as described in Section 2 for the original SPECK. After testing all sets of type $S$, a set of type $I$ is processed by testing it against the current threshold $n$. If the set is found to be significant it is divided into three subsets-one set of type $I$ and two unequal sets of type $Y$. Of the two $Y$ sets, one consists of 3 lower subbands at the lowest decomposition level in $I$, and the second consists of 4 higher subbands. Fig. 1 illustrates this partitioning scheme. It is worth mentioning that the current partitioning order is suggested by performance analysis of the algorithm on a limited number of MFM images. The optimal partitioning of $I$ sets must be investigated further. The idea behind this partitioning takes advantage of the fact that the concentration of energy decreases down the pyramid; also it is quite likely in MFM images that a set $I$ if found significant has only a single (or very few) significant coefficient(s). In addition, as mentioned in previous sections, owing to the high correlation of spectral subbands higher-frequency subbands will have fewer significant coefficient. Therefore, $Y$ subsets are created such that 3 low-frequency subbands that have a greater chance of possessing significant coefficients are coded together, while the 4 higher spectral frequency subbands are encoded together; the sparsity of significant coefficients is greater in these subbands. Sets $Y$ are tested against the current threshold $n$, and if a set is found insignificant it remains type $Y$; otherwise, it is divided into $\left|Y_{m}\right|$ subsets of type $S$. The new subsets are tested for significance like the previous $S$-type sets. 

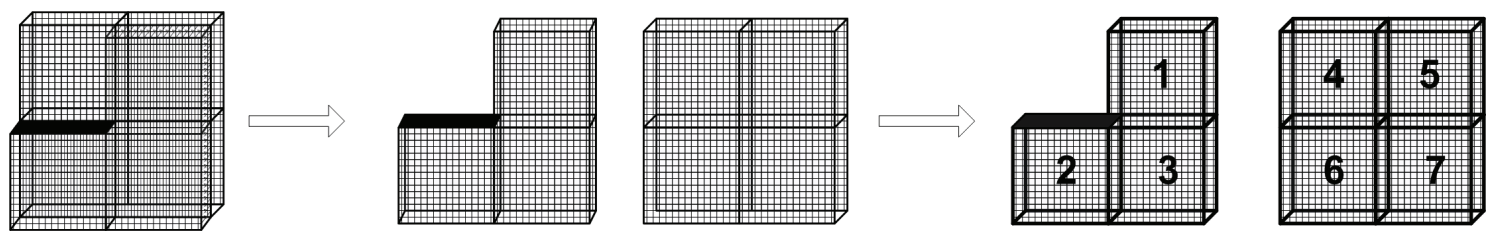

Fig. 1. Successive partitioning of significant subsets from set of type $I$ to sets of type $I$ : (from left) $I$ is the initial set, the set is than split in the middle figure into three subsets: two subsets of type $Y$ and one subset of type $I$, and finally the right-most figure shows $Y$ sets split into $S$ subsets.

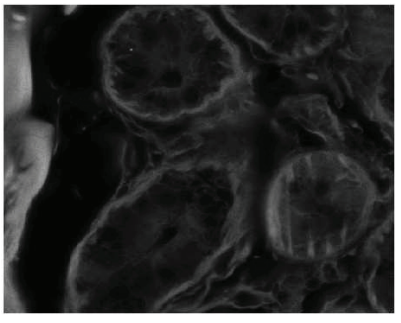

(a)

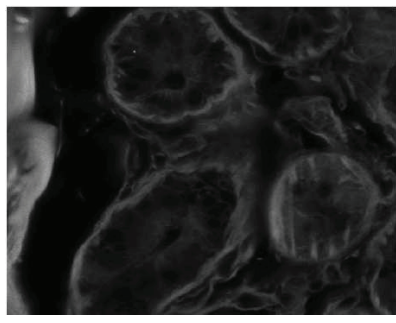

(b)

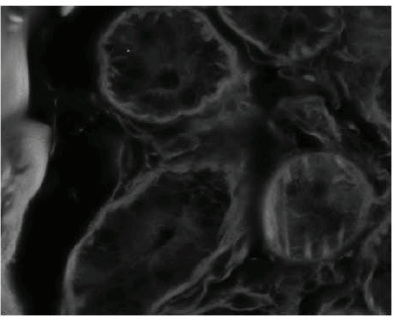

(c)

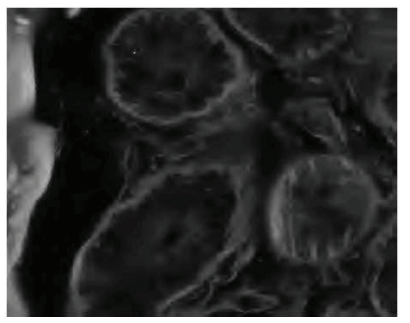

(d)

Fig. 2. (a) The sixth band of the original Cells image. (b), (c), and (d) show the same band encoded at $0.55 \mathrm{bpp}, 0.25 \mathrm{bpp}$, 0.05 bpp respectively using the proposed method (c) Coded Image at $0.25 \mathrm{bpp}$ (d) Coded Image at $0.05 \mathrm{bpp}$.

After all sets have been processed against the current threshold, the algorithm initiates the refinement pass. The threshold is lowered each time for subsequent iterations of sorting and refinement pass until the desired bit rate is achieved.

\section{NUMERICAL RESULTS}

To assess the performance of the proposed encoder, several tests have been preformed on a number of 16-band MFM images (each band was 8 bit, $512 \times 512$ pixels). We used $9 / 7$ biorthogonal filter for wavelet decomposition. Five levels of decomposition were carried out along the spatial as well as the spectral dimension. Fig. 2 highlights the performance of the proposed algorithm by comparing a single band of multispectral fluorescence image at different bit rates using the pro-

\begin{tabular}{c||c|c|c|c}
\hline \multirow{2}{*}{ Coding Method } & \multicolumn{5}{c}{ PSNR (dB) } \\
& $0.05 \mathrm{bpp}$ & $0.10 \mathrm{bpp}$ & $0.25 \mathrm{bpp}$ & $0.55 \mathrm{bpp}$ \\
\hline \hline \multicolumn{5}{c}{ Cells Image $512 \times 512 \times 8$} \\
\hline \hline Proposed Method & 22.91 & 23.92 & 31.65 & 36.31 \\
\hline 2D SPIHT & 18.27 & 20.31 & 27.61 & 32.91 \\
\hline 3D SPIHT & 23.49 & 24.91 & 29.54 & 34.33 \\
\hline 2D SPECK & 20.72 & 22.26 & 29.90 & 35.29 \\
\hline \hline \multicolumn{5}{c}{ Thymus Image $512 \times 512 \times 8$} \\
\hline Proposed Method & 23.67 & 25.81 & 29.31 & 34.93 \\
\hline 2D SPIHT & 19.09 & 21.08 & 24.20 & 27.49 \\
\hline 3D SPIHT & 24.41 & 25.33 & 27.38 & 32.33 \\
\hline 2D SPECK & 14.73 & 17.77 & 20.14 & 23.15 \\
\hline \hline \multicolumn{5}{||}{ Dish Image $512 \times 512 \times 8$} \\
\hline Proposed Method & 22.62 & 25.11 & 27.89 & 32.77 \\
\hline 2D SPIHT & 20.38 & 22.80 & 25.16 & 25.99 \\
\hline 3D SPIHT & 23.78 & 25.98 & 27.01 & 31.11 \\
\hline 2D SPECK & 19.98 & 22.75 & 24.89 & 28.86 \\
\hline
\end{tabular}

Table 1. Performance comparison against other techniques. Average PSNR over all bands is reported.

posed method. The proposed method has also been compared with related state-of-the-art methods, namely SPIHT[4], 3D SPIHT[7], and 2D SPECK[5]. The results presented in Table 1 demonstrate that the proposed algorithm outperforms contemporary SPIHT- and SPECK-based techniques in terms of bit rate per quality assessment measure (PSNR). At lower bit rates 3D SPIHT performs slightly better than the proposed method, because of the progressive encoding of coefficients in the $3 \mathrm{D}$ hierarchal tree, but the overall performance of the proposed method is much better.

\section{CONCLUSION}

A low-complexity modification of conventional waveletbased SPECK encoder, developed for compression of biological multispectral images, is proposed. The proposed concept is based on the observation that owing to the nature of multispectral fluorescence imaging, significant coefficients in higher subbands of wavelet decomposed images are sparse; therefore, encoding higher subbands as a single block while optimizing the block size will result in significant bit savings. The proposed method compares favorably with contemporary SPECK- and SPIHT-based methods both in terms of bit rate and computational complexity. 


\section{REFERENCES}

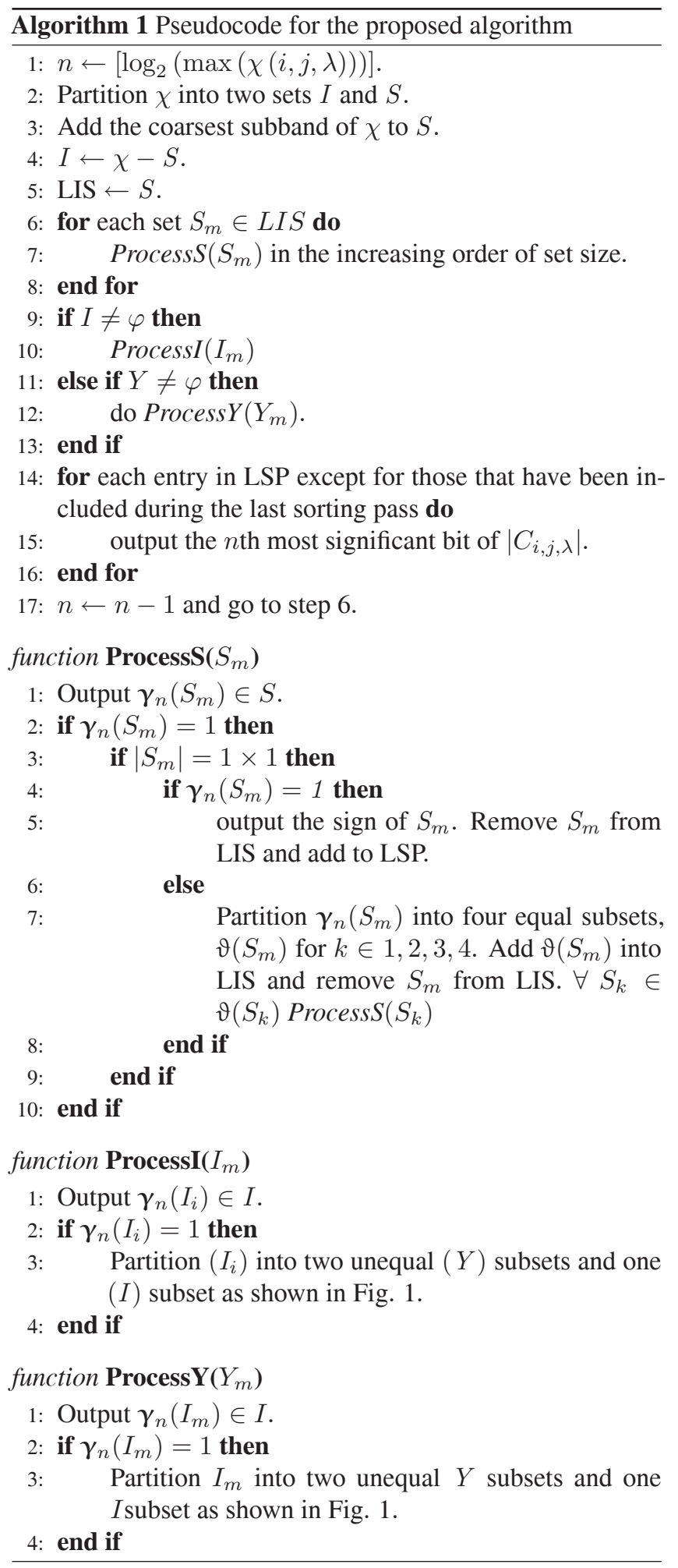

[1] W. M. Ahmed, S. J. Leavesley, B. Rajwa, M. N. Ayyaz, A. Ghafoor, and J. P. Robinson, "State of the art in information extraction and quantitative analysis for multimodality biomolecular imaging," Proceedings of the IEEE, vol. 96, no. 3, pp. 512-531, 2008.

[2] T. Bernas, E. K. Asem, J. P. Robinson, and B. Rajwa, "Compression of fluorescence microscopy images based on the signal-to-noise estimation," Microscopy Research and Technique, vol. 69, no. 1, pp. 1-9, 2006.

[3] J. M. Shapiro, "Embedded image coding using zerotrees of wavelet coefficients," Signal Processing, IEEE Transactions on, vol. 41, no. 12, pp. 3445-3462, 1993.

[4] A. Said and W. A. Pearlman, "A new, fast, and efficient image codec based on set partitioning in hierarchical trees," Circuits and Systems for Video Technology, IEEE Transactions on, vol. 6, no. 3, pp. 243-250, 1996.

[5] W. A. Pearlman, A. Islam, N. Nagaraj, and A. Said, "Efficient, low-complexity image coding with a setpartitioning embedded block coder," Circuits and Systems for Video Technology, IEEE Transactions on, vol. 14, no. 11, pp. 1219-1235, 2004.

[6] D. Taubman, "High performance scalable image compression with EBCOT," Image Processing, IEEE Transactions on, vol. 9, no. 7, pp. 1158-1170, 2000.

[7] B. Kim, Z. Xiong, and W. Pearlman, "Low bit-rate scalable video coding with 3-D set partitioning inhierarchical trees (3-D SPIHT)," IEEE Trans. on Circuits and Systems for Video Technology, vol. 10, no. 8, pp. 13741387, December 2000.

[8] P. L. Dragotti, G. Poggi, and A. R. P. Ragozini, “Compression of multispectral images by three-dimensional SPIHT algorithm," Geoscience and Remote Sensing, IEEE Transactions on, vol. 38, no. 1, pp. 416-428, 2000.

[9] X. Tang, W. A. Pearlman, and J. W. Modestino, "Hyperspectral image compression using three-dimensional wavelet coding," in Image and Video Communications and Processing 2003, Santa Clara, CA, USA, May 2003, vol. 5022, pp. 1037-1047, SPIE.

[10] F. Khelifi, F. Kurugollu, and A. Bouridane, "SPECKBased lossless multispectral image coding," Signal Processing Letters, IEEE, vol. 15, pp. 69-72, 2008. 